Strategic Information Transmission

Author(s): Vincent P. Crawford and Joel Sobel

Source: Econometrica, Vol. 50, No. 6 (Nov., 1982), pp. 1431-1451

Published by: The Econometric Society

Stable URL: http://www.jstor.org/stable/1913390

Accessed: 10/12/2010 18:32

Your use of the JSTOR archive indicates your acceptance of JSTOR's Terms and Conditions of Use, available at http://www.jstor.org/page/info/about/policies/terms.jsp. JSTOR's Terms and Conditions of Use provides, in part, that unless you have obtained prior permission, you may not download an entire issue of a journal or multiple copies of articles, and you may use content in the JSTOR archive only for your personal, non-commercial use.

Please contact the publisher regarding any further use of this work. Publisher contact information may be obtained at http://www.jstor.org/action/showPublisher?publisherCode=econosoc.

Each copy of any part of a JSTOR transmission must contain the same copyright notice that appears on the screen or printed page of such transmission.

JSTOR is a not-for-profit service that helps scholars, researchers, and students discover, use, and build upon a wide range of content in a trusted digital archive. We use information technology and tools to increase productivity and facilitate new forms of scholarship. For more information about JSTOR, please contact support@jstor.org. 


\title{
STRATEGIC INFORMATION TRANSMISSION
}

\author{
By Vincent P. CRAwFord and Joel Sobel ${ }^{1}$ \\ "Oh, what a tangled web we weave, when first we practice to deceive!" \\ -Sir Walter Scott
}

\begin{abstract}
This paper develops a model of strategic communication, in which a better-informed Sender $(S)$ sends a possibly noisy signal to a Receiver $(R)$, who then takes an action that determines the welfare of both. We characterize the set of Bayesian Nash equilibria under standard assumptions, and show that equilibrium signaling always takes a strikingly simple form, in which $S$ partitions the support of the (scalar) variable that represents his private information and introduces noise into his signal by reporting, in effect, only which element of the partition his observation actually lies in. We show under further assumptions that before $S$ observes his private information, the equilibrium whose partition has the greatest number of elements is Pareto-superior to all other equilibria, and that if agents coordinate on this equilibrium, $R$ 's equilibrium expected utility rises when agents' preferences become more similar. Since $R$ bases his choice of action on rational expectations, this establishes a sense in which equilibrium signaling is more informative when agents' preferences are more similar.
\end{abstract}

\section{INTRODUCTION}

MANY OF THE DIFFICULTIES ASSOCIATED with reaching agreements are informational. Bargainers typically have different information about preferences and even about what is feasible. Sharing information makes available better potential agreements, but it also has strategic effects that make one suspect that revealing all to an opponent is not usually the most advantageous policy. Nevertheless, it seems clear that even a completely self-interested agent will frequently find it advantageous to reveal some information. How much, and how the amount is related to the similarity of agents' interests, are the subjects of this paper.

While our primary motivations stem from the theory of bargaining, we have found it useful to approach these questions in a more abstract setting, which allows us to identify the essential prerequisites for the solution we propose. There are two agents, one of whom has private information relevant to both. The better-informed agent, henceforth called the Sender $(S)$, sends a possibly noisy

\footnotetext{
${ }^{1}$ We are grateful to Franklin Fisher, Roger Gordon, Bengt Holmström, David Kreps, Roger Myerson, Jean Tirole, anonymous referees, and participants in seminar presentations at Bell Labs, The University of California (Berkeley, Irvine, and San Diego), Caltech, Cambridge, CERAS, CORE, Leeds, LSE, Princeton, Tel Aviv, UBC, the AUTE meetings, the San Diego Summer Meetings of the Econometric Society, and a Stanford IMSSS workshop for helpful comments. We owe special thanks to Co-Editor James Mirrlees, who made numerous helpful expository criticisms of earlier drafts, insisted that a result along the lines of Theorem 2 was available (and that we prove it), and suggested the method of proof. Crawford's work was supported in part by NSF grant SES79-05550. Sobel's work was partially supported by the SSRC while he was at Nuffield College, Oxford, in connection with the project, "Incentives, Consumer Uncertainty, and Public Policy."
} 
signal, based on his private information, to the other agent, henceforth called the Receiver $(R) . R$ then makes a decision that affects the welfare of both, based on the information contained in the signal. In equilibrium, the decision rules that describe how agents choose their actions in the situations in which they find themselves are best responses to each other.

The model and its relationship to the literature are described in Section 2. Under assumptions akin to those commonly maintained in the signaling literature, equilibrium is characterized in Section 3 in a strikingly simple way. Although $S$ 's choice of signaling rule is not restricted a priori, in equilibrium he partitions the support of the probability distribution of the variable that represents his private information and, in effect, introduces noise into his signal by reporting only which element of the partition his observation actually lies in. This represents $S$ 's optimal compromise between including enough information in the signal to induce $R$ to respond to it and holding back enough so that his response is as favorable as possible.

There are, in general, several essentially different equilibria, but we argue in Sections 4 and 5 that the one whose partition has the greatest number of elements is a reasonable one for agents to coordinate on, because it is both salient and, before $S$ observes his private information, Pareto-superior to all other equilibria. Given this selection, we show under stronger assumptions that, in a sense made more precise in Sections 4 and 5, the more similar agents' preferences, the more informative the equilibrium signal.

Section 6 concludes the paper with a brief summary and suggestions for further study.

Our results have, in addition to their intrinsic interest, important implications for the design of models that relate the quality of bargaining outcomes to the bargaining environment. In particular, the rationalist explanations of the occurrence of bargaining impasses, and of the relationship of their frequency to the bargaining environment, with which we are familiar (see Chatterjee and Samuelson [1], Crawford [2], and Sobel and Takahashi [14], for example) all rest on agents having different information, either about preferences or about the extent to which they have succeeded in committing themselves to their demands. These models all abstract from the possibility that agents may find it useful to communicate other than by their demands. Our model sheds some light on when this is an innocuous simplification, and when it is likely to distort the conclusions.

Our model is also potentially applicable to many other situations where strategic communication is a possibility. Example applications include business partnerships, doctor-patient and lawyer-client relationships, and oligopoly (see Novshek and Sonnenschein [12]). Finally, it can be viewed as a principal-agent model, with $S$ the agent and $R$ the principal. As will become clear in Section 2, however, we depart from the principal-agent literature by treating the principal and the agent strategically symmetrically, in contrast to the usual treatment of the principal as a Stackelberg leader. 


\section{THE MODEL}

There are two players, a Sender $(S)$ and a Receiver $(R)$; only $S$ has private information. $S$ observes the value of a random varible, $m$, whose differentiable probability distribution function, $F(m)$, with density $f(m)$, is supported on $[0,1]$. $S$ has a twice continuously differentiable von Neumann-Morgenstern utility function $U^{S}(y, m, b)$, where $y$, a real number, is the action taken by $R$ upon receiving $S$ 's signal and $b$ is a scalar parameter we shall later use to measure how nearly agents' interests coincide. $R$ 's twice continuously differentiable von Neumann-Morgenstern utility function is denoted $U^{R}(y, m)$. All aspects of the game except $m$ are common knowledge.

Throughout the paper we shall assume that, for each $m$ and for $i=R, S$, denoting partial derivatives by subscripts in the usual way, $U_{1}^{i}(y, m)=0$ for some $y$, and $U_{11}^{i}(\cdot)<0$, so that $U^{i}$ has a unique maximum in $y$ for each given $(m, b)$ pair; and that $U_{12}^{i}(\cdot)>0$. The latter condition is a sorting condition analogous to those that appear in the signaling literature; it ensures that the best value of $y$ from a fully informed agent's standpoint is a strictly increasing function of the true value of $m$.

The game proceeds as follows. $S$ observes his "type," $m$, and then sends a signal to $R$; the signal may be random, and can be viewed as a noisy estimate of $m$. $R$ processes the information in $S$ 's signal and chooses an action, which determines players' payoffs.

The solution concept we shall employ is Harsanyi's [4] Bayesian Nash equilibrium, which is simply a Nash equilibrium in the decision rules that relate agents' actions to their information and to the situations in which they find themselves. Each agent responds optimally to his opponent's strategy choice, taking into account its implications in the light of his probabilistic beliefs, and maximizing expected utility over his possible strategy choices. Although $S$ 's signal necessarily precedes $R$ 's action in time, because $R$ observes only the signal (and not the signaling rule) $S$ 's choice of signaling rule and $R$ 's choice of action rule are strategically "simultaneous." Since we do not allow $R$ to commit himself to an action rule and communicate it before $S$ chooses his signaling rule, our solution concept differs from that employed in principal-agent models like Holmström's [6].

The Bayesian Nash equilibrium is both the natural generalization of the ordinary Nash equilibrium to games with incomplete information and a natural extension of the concept of rational-expectations equilibrium to situations where strategic interactions are important. It is, therefore, a sensible choice of equilibrium concept with which to study strategic communication, guaranteeing that in equilibrium, agents who understand the game extract all available information from signals. To put it another way, this equilibrium concept guarantees that agents' conditional probabilistic beliefs about each other's actions and characteristics are self-confirming.

Formally, an equilibrium consists of a family of signaling rules for $S$, denoted 
$q(n \mid m)$, and an action rule for $R$, denoted $y(n)$, such that: for each $m \in[0,1], \int_{N} q(n \mid m) d n=1$, where the Borel set $N$ is the set of feasible signals, and if $n^{*}$ is in the support of $q(\cdot \mid m)$, then $n^{*}$ solves $\max _{n \in N} U^{S}(y(n), m, b)$; and

$$
\begin{aligned}
& \text { for each } n, y(n) \text { solves } \max _{y} \int_{0}^{1} U^{R}(y, m) p(m \mid n) d m, \\
& \text { where } p(m \mid n) \equiv q(n \mid m) f(m) / \int_{0}^{1} q(n \mid t) f(t) d t .^{2}
\end{aligned}
$$

Condition (1) says that $S$ 's signaling rule yields an expected-utility maximizing action for each of his information "types," taking $R$ 's action rule as given. Condition (2) says that $R$ responds optimally to each possible signal, using Bayes' Rule to update his prior, taking into account $S$ 's signaling strategy and the signal he receives. Since $U_{11}^{R}(\cdot)<0$, the objective function in (2) is strictly concave in $y$; therefore, $R$ will never use mixed strategies in equilibrium.

Our model departs from the non-strategic signaling literature (see, for example, Spence [15]) principally in the nature of its signaling costs. Signaling models typically have exogenously given differential signaling costs, which allow the existence of equilibria in which agents are perfectly sorted. Our model has no such costs. But $R$ 's equilibrium choice of action rule generally creates endogenous signaling costs, which allow equilibria with partial sorting. This shows that exogenous differential signaling costs are not always needed for informative signaling.

Our model is closely related to that of Green and Stokey [3], who study strategic information transmission using a definition of equilibrium that differs

\footnotetext{
${ }^{2}$ More precisely, we may define an equilibrium to be an action rule for $R$, denoted $y(n)$, and, for $S$, a probability distribution $\hat{\mu}$ on the Borel-measurable subsets of $[0,1] \times[N]$ for which $\hat{\mu}(A \times[N])$ $=\int_{A} f$ for all measurable sets $A$. Loève [8, pp. 137-138] shows that in this setting there exist regular conditional distributions $q(\cdot \mid m)$ and $p(\cdot \mid n)$ for $(m, n) \in[0,1] \times[N]$. Then, in place of (1) we have
}

$$
\hat{\mu} \text { solves } \max _{\mu} \int_{0}^{1} \int_{N} U^{S}(y(n), m, b) d \mu,
$$

where the maximum is taken over all measures on the Borel-measurable subsets of $[0,1] \times[N]$. Since

$$
\int_{0}^{1} \int_{N} U^{S}(y(n), m, b) d \mu=\int_{0}^{1}\left[\int_{N} U^{S}(y(n), m, b) q(d n \mid m)\right] d m
$$

the conditional distributions $q(\cdot \mid m)$ satisfy (1).

Milgrom and Weber [11], who introduced this distributional approach, show that it is equivalent to the mixed strategies used in the text. In the present context, this formulation guarantees that $q(\cdot \mid \mathrm{m})$ and $p(\cdot \mid n)$ are measurable functions of $m$ and $n$ and hence that the integrals in (1) and (2) are well-defined. References to the measure $\hat{\mu}$, as well as to the fact that equalities hold almost surely, are suppressed in the text. If $S$ observes $m$ before choosing $q(n \mid m)$, the signaling rules for values of $m$ other than the true one should be viewed as a way of formalizing $R$ 's beliefs about the meaning of $S$ 's signals. 
from ours only in assuming that an agent learns his private information after his choice of strategy. We have adopted the alternative assumption that agents already know their private information when choosing their strategies, but in the present context the two definitions are equivalent. Thus, the main difference between our paper and Green and Stokey's is the question considered. They take preferences as given and study the effects of improved information on agents' welfares at equilibrium; we take information as given and study how agents use it differently when their preferences become more similar. (Holmström [6] studies the latter question in a principal-agent model.) Green and Stokey's [3] model has many equilibria, including some, which they call "partition" equilibria, in which $S$ introduces noise only by not discriminating as finely as possible in his signal among the different information states he is capable of distinguishing; they focus on these. As pointed out above, our model has multiple equilibria as well, but only partition equilibria. This difference arises because of our additional restrictions on preferences.

Our model is also related to those of Kreps and Wilson [7] and Milgrom and Roberts $[9,10]$, who handle the problem of information transmission in the same way we do. Milgrom and Roberts' [9] model is closest in form to ours; but they focus mainly on perfectly informative equilibria. This precludes the study of the optimal amount of noise to include in a signal. Perfectly informative equilibria do not exist in our model, mainly because we assume that signaling has no cost to $S$ other than that inherent in its effect on $R$ 's choice of action.

\section{EQUILIBRIUM}

This section establishes the existence of equilibria in our model, and characterizes them. It is shown that all equilibria are partition equilibria, in which, in effect, $S$ introduces noise into his signal only by not discriminating as finely as possible among the information states he can distinguish. Further, we show that if $R$ 's and $S$ 's preferences differ, there is a finite upper bound, denoted $N(b)$, on the "size" (that is, the number of subintervals) of an equilibrium partition; and that there exists at least one equilibrium of each size from one through $N(b)$. Necessary and sufficient conditions for a partition of a given size to be consistent with equilibrium are given. In Sections 4 and 5, we give conditions that guarantee uniqueness of equilibrium for each size, and argue that agents might reasonably be expected to coordinate on the equilibrium of size $N(b)$.

We shall defer, for the sake of exposition, consideration of the form of the equilibrium signaling rules, and begin by considering the structure of the set of actions that, in equilibrium, are chosen by $R$ with positive prior probability.

Let $\bar{N} \equiv\{n: y(n)=\bar{y}\}$. We say that an action $\bar{y}$ is induced by an $S$-type $\bar{m}$ if $\int_{\bar{N}} q(n \mid \bar{m}) d n>0$. Notice that if $Y$ is the set of all actions induced by some $S$-type, then if $\bar{m}$ induces $\bar{y}, U^{S}(\bar{y}, \bar{m}, b)=\max _{y \in Y} U^{S}(y, \bar{m}, b)$. (We assume without loss of generality that $R$ takes actions in $Y$ for values of $n$ not in the support of any $q(\cdot \mid m)$.) Since $U_{11}^{S}(\cdot)<0, U^{S}(y, m, b)$ can take on a given value for at most two values of $y$. Thus, $\bar{m}$ can induce no more than two actions in 
equilibrium. Define, for all $m \in[0,1]$,

$$
y^{S}(m, b) \equiv \arg \max U^{S}(y, m, b)
$$

and

$$
y^{R}(m) \equiv \arg \max U^{R}(y, m),
$$

where $\arg \max U^{S}(y, m, b)$, for example, denotes the value of $y$ that maximizes $U^{S}(y, m, b)$. Since $U_{11}^{i}(\cdot)<0$ and $U_{12}^{i}(\cdot)>0, i=R, S, y^{S}(m, b)$ and $y^{R}(m)$ are well defined and continuous in $m$.

LEMMA 1: If $y^{S}(m, b) \neq y^{R}(m)$ for all $m$, then there exists an $\epsilon>0$ such that if $u$ and $v$ are actions induced in equilibrium, $|u-v| \geq \epsilon$. Further, the set of actions induced in equilibrium is finite.

Proof: Let $u$ and $v$, with $u<v$, be two actions induced in equilibrium. Since an $S$-type who induces $u(v)$ thereby reveals a weak preference for that action over $v(u)$, by continuity there exists an $\bar{m} \in[0,1]$ such that $U^{S}(u, \bar{m}, b)=$ $U^{S}(v, \bar{m}, b)$. Since $U_{11}^{S}(\cdot)<0$ and $U_{12}^{S}(\cdot)>0$, it follows from this that

$$
\begin{aligned}
& u<y^{S}(\bar{m}, b)<v, \\
& u \text { is not induced by any } S \text {-type } m>\bar{m} \text {, and } \\
& v \text { is not induced by any } S \text {-type } m<\bar{m} .
\end{aligned}
$$

In turn, (6), (7), and our assumption that $U_{12}^{R}(\cdot)>0$ imply that

$$
u \leq y^{R}(\bar{m}) \leq v .
$$

However, if $y^{R}(m) \neq y^{S}(m, b)$ for all $m \in[0,1]$, there is an $\epsilon>0$ such that $\left|y^{R}(m)-y^{S}(m, b)\right| \geq \epsilon$ for all $m \in[0,1]$. It follows from (5) and (8) that $v-u$ $\geq \epsilon$. Since the set of actions induced in equilibrium is bounded by $y^{R}(0)$ and $y^{R}(1)$ because $U_{12}^{R}(\cdot)>0$, this completes the proof.

REMARKs: Lemma 1 establishes that, under our assumptions, equilibrium must involve noisy signaling unless agents' interests coincide. Because signaling is a purely informational activity in our model, it cannot be perfectly invertible and informative, as it is, for example, in the principal equilibria of Milgrom and Roberts [9]. The argument of Lemma $1 \mathrm{can}$ be used to establish that if $U_{12}^{S}$ and $U_{12}^{R}$ are one-signed, but have opposite signs, then only one action can be induced in equilibrium. Thus in this case no information is transmitted.

We shall now argue that when agents' interests differ, all equilibria in our model are partition equilibria of a particular kind. First, some notation for describing partition equilibria is needed. Let $a(N) \equiv\left(a_{0}(N), \ldots, a_{N}(N)\right)$ denote a partition of $[0,1]$ with $N$ steps and dividing points between steps $a_{0}(N), \ldots$, $a_{N}(N)$, where $0=a_{0}(N)<a_{1}(N)<\cdots<a_{N}(N)=1$. Whenever it can be done without loss of clarity in what follows, we shall write $a$ or $a_{i}$ instead of $a(N)$ or 
$a_{i}(N)$. Define, for all $\underline{a}, \bar{a} \in[0,1], \underline{a} \leq \bar{a}$,

$$
\bar{y}(\underline{a}, \bar{a}) \equiv \begin{cases}\arg \max \int_{\underline{a}}^{\bar{a}} U^{R}(y, m) f(m) d m & \text { if } \quad \underline{a}<\bar{a}, \\ y^{R}(\underline{a}) & \text { if } \quad \underline{a}=\bar{a} .\end{cases}
$$

Now we are ready to state Theorem 1, which establishes the existence of equilibria, and characterizes them.

THEOREM 1: Suppose $b$ is such that $y^{S}(m, b) \neq y^{R}(m)$ for all $m$. Then there exists a positive integer $N(b)$ such that, for every $N$ with $1 \leq N \leq N(b)$, there exists at least one equilibrium $(y(n), q(n \mid m))$, where $q(n \mid m)$ is uniform, supported on $\left[a_{i}, a_{i+1}\right]$ if $m \in\left(a_{i}, a_{i+1}\right)$,

$$
\begin{array}{r}
U^{S}\left(\bar{y}\left(a_{i}, a_{i+1}\right), a_{i}, b\right)-U^{S}\left(\bar{y}\left(a_{i-1}, a_{i}\right), a_{i}, b\right)=0 \\
\quad(i=1, \ldots, N-1),
\end{array}
$$

Further, any equilibrium is essentially ${ }^{3}$ equivalent to one in this class, for some value of $N$ with $1 \leq N \leq N(b)$.

REMARKs: Theorem 1 establishes the existence of a partition equilibrium of every size from one (completely uninformative) to $N(b)$ (the most informative, in a sense made precise below), where $N(b)$ is determined by $b$, the preferencesimilarity parameter. If preferences are identical for some value of $b$, or if $y^{S}(m, b)=y^{R}(m)$ for some $m$, existence is easily established, but finiteness does not hold in general.

Proof: The outline of the proof is as follows. Given Lemma 1, each $S$-type must, in an equilibrium of size $N$, choose from a set of $N$ values of $y$. Since $U_{12}^{S}(\cdot)>0$, the $S$-types for whom each value of $y$ that occurs in equilibrium is best form an interval, and these intervals form a partition of $[0,1]$. The partition, $a$, is determined by (A), a well-defined second-order nonlinear difference equation in the $a_{i}$, and (11) and (12), its initial and terminal conditions. Equation (A) is an "arbitrage" condition, requiring that $S$-types who fall on the boundaries between steps are indifferent between the associated values of $y$. With our assumptions on $U^{S}$, this condition is necessary and sufficient for $S$ 's signaling rule to be a best response to $y(n)$. Finally, given the signaling rules in the

\footnotetext{
${ }^{3}$ By "essentially," we mean that all equilibria have relationships between $m$ and $R$ 's induced choice of $y$ that are the same as those in the class described in the Theorem; they are, therefore, economically equivalent.
} 
statement of the Theorem, it is easily verified that the integral on the right-hand side of (9) is $R$ 's expected utility conditional on hearing a signal in the step $(\underline{a}, \bar{a})$. It follows that (10) gives $R$ 's unique best response to a signal in $\left(a_{i}, a_{i+1}\right)$, and that the signaling rules given in the Theorem are in equilibrium. Any other signaling rules that induce the same actions would also work $;^{4}$ and we close by arguing that any other signaling rules consistent with an equilibrium of a given size must, given Lemma 1 , induce the same actions.

Formally, we begin by showing that (A), (11), and (12) form a well-defined difference equation, that it has a solution for any $N$ such that $1 \leq N \leq N(b)$, and that any solution, $a$, together with the signaling rules given in the Theorem, is a best response for $S$ to the $y(n)$ that satisfies (10) for the same $a$. In the rest of this section we sometimes suppress the dependence of $U^{S}$ on $b$ for notational clarity.

First, note that, by (9) and our assumption that $U_{12}^{R}(\cdot)>0, \bar{y}\left(a_{i}, a_{i+1}\right)$ must be strictly increasing in both of its arguments. Let $a^{i}$ denote the partial partition $a_{0}, \ldots, a_{i}$, which is strictly increasing and satisfies (A). There can be at most one value of $a_{i+1}>a_{i}$ satisfying (A), because $U_{11}^{S}(\cdot)<0$ and $\bar{y}(\cdot)$ is monotonic. Thus any history $a_{0}, \ldots, a_{i}$ determines at most one relevant $a_{i+1}>a_{i}{ }^{5}$

Let

$$
K(a) \equiv \max \left\{i \text { : there exists } 0<a<a_{2}<\cdots<a_{i} \leq 1 \text { satisfying (A) }\right\} .
$$

When $y^{S}(m, b) \neq y^{R}(m)$ for every $m$, it follows from Lemma 1 that $\bar{y}\left(a_{i}, a_{i+1}\right)-$ $\bar{y}\left(a_{i-1}, a_{i}\right) \geq \epsilon$ for some $\epsilon>0$; hence $a_{i+2}-a_{i}$ is bounded above zero for any solution of $(\mathrm{A})$. Thus $K(a)$ is finite, well defined, and uniformly bounded, so $\sup _{0<a \leq 1} K(a)$ is achieved for some $\bar{a} \in(0,1]$. Let $N(b) \equiv K(\bar{a})<\infty$. It remains to show that for each $N$ such that $1 \leq N \leq N(b)$, there is a partition $a$ satisfying (A), (11), and (12). Let $a^{K(a)}$ be the partial partition of length $K(a)$ that satisfies (A) and $a_{1}^{K(a)}=a$. Since solutions to (A) vary continuously with respect to initial conditions, if $a_{K(a)}^{K(a)}$ (the last term in the partial partition $a^{K(a)}$ ) is less than unity, $K(\cdot)$ is continuous (and therefore locally constant) at $a$; moreover, $K(a)$ can change by at most one at a discontinuity. Finally, $K(1)=1$, so $K(a)$ takes on all integer values between one and $N(b)$. If $K\left(a_{1}\right)=N$ and $K(a)$ is discontinuous at $a=a_{1}$, then $a$ satisfies (A), (11), and (12).

Now we shall argue that if $a$ satisfies (A), (11), and (12), any signal in $\left(a_{i}, a_{i+1}\right)$ is a best response for an $S$ of type $m \in\left(a_{i}, a_{i+1}\right)$ to the $y(n)$ given by (10). More

\footnotetext{
${ }^{4}$ In particular, there is a pure-strategy equilibrium in which the $S$-types within each step send a given signal that differs from those sent by other $S$-types. To support an equilibrium described this way, it is necessary to include, as part of the equilibrium, a specification of how $R$ interprets signals that are not in the support of the signaling rule used by some $S$-type in equilibrium. In the present context, any such specification that does not expand the set of $R$ 's best-response actions will do.

${ }^{5} \mathrm{We}$ are able to restrict attention to the strictly increasing partitions that satisfy (A) because $U_{11}^{S}(\cdot)<0$ and the monotonicity of $\bar{y}(\cdot)$ ensure that the only nondecreasing solutions, $a$, to (A), (11), and (12) satisfy $a_{i}<a_{i+1}$ unless $a_{i}=0$ or $a_{i+1}=1$. In the latter two cases, an extreme $S$-type is indifferent between perfectly revealing his type and sending a signal in the adjacent step. Because $m=0$ and $m=1$ occur only with zero probability from $R$ 's standpoint, these equilibria are therefore essentially equivalent to those in which the extreme $S$-type does not reveal himself.
} 
precisely, (A) implies that

$$
U^{S}\left(\bar{y}\left(a_{i}, a_{i+1}\right), m\right)=\max _{j} U^{S}\left(\bar{y}\left(a_{j}, a_{j+1}\right), m\right) \text { for all } m \in\left[a_{i}, a_{i+1}\right],
$$

where the maximum in (13) is taken over $j=0, \ldots, N-1$. To see this, note that because $U_{11}^{S}(\cdot)<0$ and $\bar{y}\left(a_{i}, a_{i+1}\right)>\bar{y}\left(a_{i-1}, a_{i}\right)$, (A) implies (13) for $m=a_{i}$. Since $U_{12}^{S}(\cdot)>0$ and $m \in\left[a_{i}, a_{i+1}\right]$,

$$
\begin{aligned}
& U^{S}\left(\bar{y}\left(a_{i}, a_{i+1}\right), m\right)-U^{S}\left(\bar{y}\left(a_{k}, a_{k+1}\right), m\right) \\
& \quad \geq U^{S}\left(\bar{y}\left(a_{i}, a_{i+1}\right), a_{i}\right)-U^{S}\left(\bar{y}\left(a_{k}, a_{k+1}\right), a_{i}\right) \geq 0 \text { and } \\
& U^{S}\left(\bar{y}\left(a_{i}, a_{i+1}\right), m\right)-U^{S}\left(\bar{y}\left(a_{j}, a_{j+1}\right), m\right) \\
& \quad \geq U^{S}\left(\bar{y}\left(a_{i}, a_{i+1}\right), a_{i+1}\right)-U^{S}\left(\bar{y}\left(a_{j}, a_{j+1}\right), a_{i+1}\right) \geq 0,
\end{aligned}
$$

where (14) and (15) hold for any $0 \leq k \leq i \leq j \leq N$ and $m \in\left[a_{i}, a_{i+1}\right]$. Conversely, it is clear from this argument that, except for $S$-types who fall on the boundaries between steps, only signals of this kind are best responses for $S$.

Now consider $R$. Provided that $S$ 's signaling rule is chosen to be uniform as in the statement of the Theorem, when $R$ hears a signal in the step $\left(a_{i}, a_{i+1}\right)$

$$
p(m \mid n) \equiv q(n \mid m) f(m) / \int_{a_{i}}^{a_{i+1}} q(n \mid t) f(t) d t=f(m) / \int_{a_{i}}^{a_{i+1}} f(t) d t .
$$

Thus his conditional expected utility is

$$
\int_{a_{i}}^{a_{i+1}} U^{R}(y, m) p(m \mid n) d m=\int_{a_{i}}^{a_{i+1}} U^{R}(y, m) f(m) d m / \int_{a_{i}}^{a_{i+1}} f(t) d t .
$$

Therefore, $\bar{y}\left(a_{i}, a_{i+1}\right)$ as defined in (9) is a best response for $R$ to a signal $n \in\left(a_{i}, a_{i+1}\right)$.

Conversely, Lemma 1 shows that any equilibrium is a partition equilibrium, and the above arguments show that any equilibrium partition, $a$, must satisfy (A), (11), and (12) for some value of $N$ between unity and $N(b)$. Let $y_{i}$ be the action induced by an $S$-type $m \in\left(a_{i}, a_{i+1}\right)$ and let $N_{i} \equiv\left\{n: y(n)=y_{i}\right\}$; if $R$ hears a signal $n \in N_{i}$ in such an equilibrium, his conditional expected utility is proportional to $\int_{a_{t}+}^{a_{+}} U^{R}(y(n), m) q(n \mid m) f(m) d m$. Since $y_{i}$ is a best response to any signal $n \in N_{i}$, it must also maximize

$$
\int_{a_{i}}^{a_{i+1}} \int_{N_{t}} U^{R}(y(n), m) q(n \mid m) f(m) d n d m \equiv \int_{a_{i}}^{a_{t+1}} U^{R}(y(n), m) f(m) d m,
$$

where the identity follows because $y(n)$ is constant over the range of integration and conditional densities integrate to unity. It follows that all equilibria are essentially equivalent to those with uniform signaling rules, as given in the statement of the Theorem.

Q.E.D.

For $0 \leq a_{i-1} \leq a_{i} \leq a_{i+1} \leq 1$, let

$$
V\left(a_{i-1}, a_{i}, a_{i+1}, b\right) \equiv U^{S}\left(\bar{y}\left(a_{i}, a_{i+1}\right), a_{i}, b\right)-U^{S}\left(\bar{y}\left(a_{i-1}, a_{i}\right), a_{i}, b\right) .
$$


$V(\cdot)$ is the difference in utility to $S$-type $a_{i}$ between $\bar{y}\left(a_{i}, a_{i+1}\right)$ and $\bar{y}\left(a_{i-1}, a_{i}\right)$.

The following Lemma establishes properties of $V$ that are useful in proving Corollary 1 and in the analysis of Section 5.

LEMMA 2: If $V\left(a_{i-1}, a_{i}, a_{i+1}, b\right)=0$ for $0 \leq a_{i-1}<a_{i}<a_{i+1} \leq 1$, then $U_{1}^{S}\left(\bar{y}\left(a, a_{i}\right), a_{i}, b\right)>0$ and $V_{1}\left(a, a_{i}, a_{i+1}, b\right)<0$ for all $a \in\left[0, a_{i-1}\right]$, and $U_{1}^{S}\left(\bar{y}\left(a_{i}\right.\right.$, $\left.a), a_{i}, b\right)<0$ and $V_{3}\left(a_{i-1}, a_{i}, a, b\right)<0$ for all $a \in\left[a_{i+1}, 1\right]$.

Proof: Since $U^{S}\left(\bar{y}\left(a_{i-1}, a_{i}\right), a_{i}, b\right)=U^{S}\left(\bar{y}\left(a_{i}, a_{i+1}\right), a_{i}, b\right)$ by hypothesis, $\bar{y}\left(a_{i}\right.$, $\left.a_{i+1}\right)>\bar{y}\left(a_{i-1}, a_{i}\right)$, and $U_{11}^{S}(\cdot)<0, U_{1}^{S}\left(y, a_{i}, b\right)>0$ for $y \leq \bar{y}\left(a_{i-1}, a_{i}\right)$ and $U_{1}^{S}(y$, $\left.a_{i}, b\right)<0$ for $y \geq \bar{y}\left(a_{i}, a_{i+1}\right)$. The Lemma follows from the definition of $V$ because $\bar{y}(\cdot)$ is strictly increasing in both of its arguments.

Q.E.D.

The next result provides a simple condition on preferences that guarantees they are far enough apart so that the only equilibrium is totally uninformative.

Corollary 1: If $V(0, a, 1, b)>0$ for all $a \in[0,1]$, then $N(b)=1$; that is, the only equilibrium is uninformative.

REMARKS: If $y^{S}(a, b)>y^{R}(a)$ for all $a$, as we assume in Section 5 , then $V(0, a, 1, b)>0$ for all sufficiently large values of $a$. This is because if $y^{S}(a, b)$ $\geq y^{R}(1)$ then an $S$ of type $a$ wishes to induce $R$ to take as large an action as possible. In particular, if $y^{S}(0, b) \geq y^{R}(1)$ then $N(b)=1$. Under the monotonicity condition, (M), we shall impose in the comparative statics analysis of Section 5 , the condition of the Corollary is equivalent to $U^{S}(\bar{y}(0,1), 0, b)>U^{S}(\bar{y}(0,0)$, $0, b)$. This means that an $S$ of type $m=0$ would rather be completely disguised than perfectly revealed.

Proof: It follows from Lemma 2 that if $V\left(0, a_{1}, a_{2}, b\right)=0$ for some $0<a_{1}$ $<a_{2} \leq 1$, then $V\left(0, a_{1}, 1, b\right) \leq 0$. Hence $V(0, a, 1, b)>0$ for all $a \in[0,1]$ implies that there is no partition equilibrium of size two. Thus, by Theorem $1, N(b)=1$.

Q.E.D.

\section{AN EXAMPLE}

This section works out a simple example, to serve as an antidote to the abstractness of the previous section and an introduction to the comparative statics questions we shall ask in the next section. In the example, $F(m)$ is uniform (on $[0,1]), U^{S}(y, m, b) \equiv-(y-(m+b))^{2}$, where $b>0$ without loss of generality, and $U^{R}(y, m) \equiv-(y-m)^{2}$. These specifications satisfy all of our maintained assumptions, and have a convenient certainty-equivalence property.

Consider the conditions that characterize a partition equilibrium of size $N$. Letting $a$ denote the partition as before, we can compute

$$
\bar{y}\left(a_{i}, a_{i+1}\right)=\left(a_{i}+a_{i+1}\right) / 2, \quad i=0, \ldots, N-1 .
$$


The arbitrage condition (A) specializes to

$$
-\left(\frac{a_{i}+a_{i+1}}{2}-a_{i}-b\right)^{2}=-\left(\frac{a_{i-1}+a_{i}}{2}-a_{i}-b\right)^{2}
$$

$$
(i=1, \ldots, N-1)
$$

which can only hold, given the monotonicity of $a$, if

$$
a_{i+1}=2 a_{i}-a_{i-1}+4 b \quad(i=1, \ldots, N-1) .
$$

This second-order linear difference equation has a class of solutions parametrized by $a_{1}$ (given that $a_{0}=0$ ):

$$
a_{i}=a_{1} i+2 i(i-1) b \quad(i=1, \ldots, N) .
$$

$N(b)$ in Theorem 1 is the largest positive integer $i$ such that $2 i(i-1) b<1$, which is easily shown to be

$$
\left\langle-\frac{1}{2}+\frac{1}{2}\left(1+\frac{2}{b}\right)^{1 / 2}\right\rangle
$$

(where $\langle z\rangle$ denotes the smallest integer greater than or equal to $z$ ). Thus, it is clear that the closer $b$ approaches zero-the more nearly agents' interests coincide - the finer partition equilibria there can be. (We use "finer" informally, not in the sense of information theory.) As $b \rightarrow \infty, N(b)$ eventually falls to unity, and only the completely uninformative equilibrium remains; in fact, this occurs in our example as soon as $b$ exceeds $1 / 4$, as predicted by Corollary 1 .

It is natural to ask which of these equilibria is best for $R$ and $S$. In general, the answer ex post will be different for different values of $m$; but ex ante, the answer is simple. If $\sigma_{m}^{2}$ denotes the residual variance of $m R$ expects to have after hearing the equilibrium signal, it is easy to verify that $R$ 's and $S$ 's ex ante expected utilities are given by $E U^{R}=-\sigma_{m}^{2}$ and $E U^{S}=-\left(\sigma_{m}^{2}+b^{2}\right)$. These expressions reflect the facts that quadratic loss equals variance plus the square of bias and that the rational-expectations character of Bayesian Nash equilibrium eliminates all unconditional bias from $R$ 's interpretation of $S$ 's signal. $R$ 's desire to set $y$ at a level $b$ units lower than $S$ would prefer appears as a bias from $S$ 's standpoint. As the expressions for $E U^{R}$ and $E U^{S}$ make clear, before $S$ learns his type, he has an incentive to join with $R$ in reducing variance as much as possible.

Using (22) and substituting for the value of $a_{1},(1-2 N(N-1) b) / N$, determined by $a_{N}=1$ yields

$$
a_{i}=\frac{i}{N}+2 b i(i-N)
$$

$$
(i=0, \ldots, N)
$$

and

$$
a_{i}-a_{i-1}=\frac{1}{N}+2 b(2 i-N-1)
$$


It follows that

$$
\begin{aligned}
\sigma_{m}^{2} & =\sum_{i=1}^{N} \int_{a_{t-1}}^{a_{i}}\left[m-\frac{a_{i-1}+a_{i}}{2}\right]^{2} d m=\frac{1}{12} \sum_{i=1}^{N}\left(a_{i}-a_{i-1}\right)^{3} \\
& =\frac{1}{12} \sum_{i=1}^{N}\left[\frac{1}{N}+2 b(2 i-N-1)\right]^{3}=\frac{1}{12 N^{2}}+\frac{b^{2}\left(N^{2}-1\right)}{3} .
\end{aligned}
$$

For a given value of $N, \sigma_{m}^{2}$ is a convex function of $N$ positive terms that sum to unity, so moving the terms closer together always reduces $\sigma_{m}^{2}$. Letting $b$ approach zero clearly does this, and $\sigma_{m}^{2}$ is plainly minimized, for given $N$, when $b=0$, since only then are all terms equalized. The expression in (25) can be used to show that for a given value of $b$, the partition equilibrium of size $N(b)$ (the largest possible) minimizes $\sigma_{m}^{2}$, and is therefore ex ante Pareto-superior to all other equilibria. Since we shall later prove a generalization of this, these calculations are not reproduced here.

While it must be admitted that comparative statics is a risky business when there are multiple equilibria, we view these results as tending to confirm our intuition that equilibrium should involve more informative signaling the closer agents' interests. There are two reasons for this. First, for a partition of given size, letting $b$ approach zero reduces the equilibrium variance in our example. And second, letting $b$ approach zero, when it expands the set of sizes of partition equilibria that can exist, always does so in the direction of making possible equilibria with "finer" partitions, and therefore lower variances. Because $F$ is fixed and $R$ bases his choice of $y$ on rational expectations, it is natural to take his expected utility as a measure of informativeness. In the quadratic case, $E U^{R}$ $=-\sigma_{m}^{2}$, so if jumps from one size of partition to another occur, if at all, only in the direction in which the set of equilibria expands, our intuition about comparative statics will be fully borne out. These conclusions suggest that it might be useful to seek more general conditions under which making preferences more similar shifts the set of equilibria in a more informative direction; we do this in the next section.

But first, we would like to consider, in the relatively simple context of our example, whether complete agnosticism about which equilibrium will occur is justified, or if some can be ruled out by making further plausible assumptions. Two promising avenues of this type seem open to us. The first is to apply Schelling's [13, Chapter 4] idea of seeking equilibria that seem "prominent," in the hope that they might serve as "focal points" to help agents coordinate their strategy choices. It seems clear to us that in our model, the coarsest and the finest partition equilibria for a given value of $b$ are prominent. The coarsest one, which is necessarily totally uninformative, does not seem very sensible to us (partly for efficiency reasons discussed below), so there remains a case for the equilibrium with $N=N(b)$.

The second avenue is to apply Harsanyi's [5, Chapter 7] suggestion that only equilibria that are not Pareto-inferior to other equilibria are likely to be observed. The idea here is that if the possibilities for enforcing agreements have been properly included in the specification of the game, only equilibria are really 
enforceable. But within the set of equilibria, the usual ceteris paribus tendency for efficient outcomes to prevail in economic situations should remain. If agent $S$ learns his type before he has an opportunity to reach an agreement with $R$ about coordinating strategy choices, there is little to be gained from this approach. As we shall see shortly, different $S$-types have quite different desires about which equilibrium should occur, and it would therefore be quite difficult for an $S$ who knew his type to negotiate about a selection from the equilibrium correspondence without revealing information about his type beyond that contained in his signal (and thereby vitiating our characterization of equilibrium). But if the selection agreement is made ex ante for a single play of the game, or if it is viewed as a convention evolved over repeated play against different opponents, a strong case can again be made, in the example, for the equilibrium with the finest partition: $N=N(b)$. This leads to the comparative statics results we hoped to establish. The arguments of the next section show that this case for the finest partition equilibrium remains intact under a reasonable assumption that is satisfied in our example but goes considerably beyond it. We conclude that the problems inevitably associated with multiple equilibria are particularly mild here.

Continuing the analysis of the example, consider the case $b=1 / 20$. Then $N(b)=\langle-1 / 2+(1 / 2) \sqrt{41}\rangle=3$, and there are three partition equilibria: $K=1$, with $a_{0}(1)=0$ and $a_{1}(1)=1 ; K=2$, with $a_{0}(2)=0, a_{1}(2)=2 / 5$, and $a_{2}(2)=1$; and $K=3$, with $a_{0}(3)=0, a_{1}(3)=2 / 15, a_{2}(3)=7 / 15$, and $a_{3}(3)=1$. The reader can easily verify that for $K=1$, the utility of an $S$ of type $m$ (who faces no uncertainty) is $-((9 / 20)-m)^{2}$; for $K=2$, it is $-((3 / 20)-m)^{2}$ if $m \in[0,2 / 5)$ and $-((13 / 20)-m)^{2}$ if $m \in(2 / 5,1]$; and for $K=3$, it is $-((1 / 60)-m)^{2}$ if $m \in[0,2 / 15),-((1 / 4)-m)^{2}$ if $m \in(2 / 15,7 / 15)$, and $-((41 / 60)-m)^{2}$ if $m$ $\in(7 / 15,1]$. These imply, as Figure 1 shows, that $K=1$ is best for $m \in(7 / 20$,

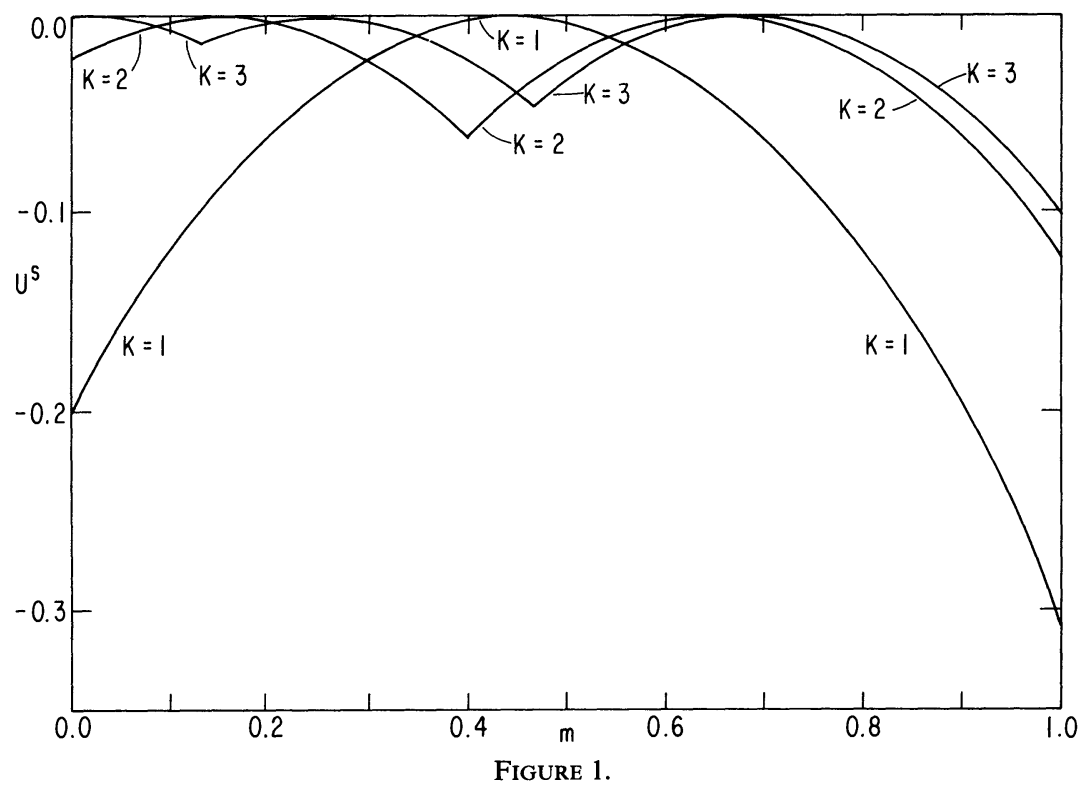


$11 / 20) ; K=2$ is best for $m \in(1 / 12,1 / 5)$ and $m \in(11 / 20,2 / 3)$; and $K=3$ is best for $m \in[0,1 / 12), m \in(1 / 5,7 / 20)$, and $m \in(2 / 3,1]$. Different $S$-types generally prefer different equilibria.

Before moving on to the more general analysis of the next section, consider how the equilibrium payoffs of the $S$-types in the equilibria just characterized compare with the payoffs that would result from the truth, if it were believed by $R$. (The truth always yields $R$ a payoff of zero.) The reader can verify that $S$ 's equilibrium payoff is as good or better than truth-telling, which yields him $-b^{2}=-1 / 400$, if and only if (barring ties): $m \in(2 / 5,1 / 2)$ when $K=1$; $m \in(1 / 10,1 / 5)$ or $m \in(3 / 5,7 / 10)$ when $K=2$; and $m \in[0,1 / 15), m \in(1 / 5$, $3 / 10)$, or $m \in(19 / 30,11 / 15)$ when $K=3$. It is therefore frequently true that a commitment to tell the truth would, if believed, pay off. (In the example, such commitments are always beneficial ex ante, since they raise $E U^{S}$ from $-\left(b^{2}+\right.$ $\left.\sigma_{m}^{2}\right)$ to $-b^{2}$; we have been unable to verify whether this is true under more general conditions.) In our model, such commitments are impossible because they are unenforceable: $R$ would interpret a true signal incorrectly because he is aware of the incentives for $S$ to lie, and $S$ cannot, within the confines of our game, remove these incentives. Even though he would like to tell the truth, he is forced to cut his losses by lying as the equilibrium in force dictates. This result is strongly reminiscent of the main result in Milgrom and Roberts [9].

\section{COMPARATIVE STATICS}

It is natural to ask at this point to what extent the strong comparative statics results that hold in our example can be generalized beyond the specifications used in Section 4. While we cannot offer a complete answer to this question at present, this section provides more general sufficient conditions to establish that the results are not merely artifacts of our choice of example.

Recall that $V\left(a_{i-1}, a_{i}, a_{i+1}, b\right) \equiv U^{S}\left(\bar{y}\left(a_{i}, a_{i+1}\right), a_{i}, b\right)-U^{S}\left(\bar{y}\left(a_{i-1}, a_{i}\right), a_{i}, b\right)$. It will be assumed throughout this section that $U^{S}(y, m, 0) \equiv U^{R}(y, m)$, that $b \geq 0$, and that $U_{13}^{S}(\cdot)>0$ everywhere. These assumptions guarantee that $V_{4}(\cdot)>0$ and that $y^{S}(m, b)>y^{R}(m)$ for all $b>0$; they are satisfied in our examples. That $U_{13}^{S}(\cdot)>0$ means that an increase in $b$ shifts $S$ 's preferences away from $R$ 's for all values of $m$. For a fixed value of $b$, we shall call a sequence $\left\{a_{0}, \ldots, a_{N}\right\}$ a forward (backward) solution to (A) if $V\left(a_{i-1}, a_{i}, a_{i+1}, b\right)=0$ for $0<i<N$ and $a_{0}<a_{1}\left(a_{0}>a_{1}\right)$. We shall impose in addition the following monotonicity condition on solutions of $(\mathrm{A})$ :

(M) For a given value of $b$, if $\hat{a}$ and $\tilde{a}$ are two forward solutions of (A) with $\hat{a}_{0}=\tilde{a}_{0}$ and $\hat{a}_{1}>\tilde{a}_{1}$, then $\hat{a}_{i}>\tilde{a}_{i}$ for all $i \geq 2$.

At times it will be convenient to use the following equivalent form of (M):

$\left(\mathbf{M}^{\prime}\right)$ For a given value of $b$, if $\hat{a}$ and $\tilde{a}$ are two backward solutions of (A) with $\hat{a}_{0}=\tilde{a}_{0}$ and $\hat{a}_{1}>\tilde{a}_{1}$, then $\hat{a}_{i}>\tilde{a}_{i}$ for all $i \geq 2$. 
Assumption (M) requires that for a given value of $b$, starting from any $a_{0} \in[0,1]$, the economically relevant solutions of (A) must all move up or down together. It therefore guarantees that the boundary-value problem defined by (A), (11), and (12) has at most one solution for fixed $N$, and thus enables us to compare partitions of fixed size as $b$ varies. It is immediately clear from (22) that (M) is always satisfied in our example and that it is robust at least to small deviations from our example. Theorem 2 provides conditions on priors and preferences that imply $(\mathbf{M})$ :

THEOREM 2: For a given value of $b$, if $U_{2}^{S}(y, a, b)+U_{1}^{S}(y, a, b)$ is nondecreasing in $y$ and $\int_{0}^{a} U_{11}^{R}(y, m) f(m) d m+U_{1}^{R}(y, a) f(a)$ is nonincreasing in a, then all solutions to (A) satisfy condition (M).

Proof: Let $a_{0} \in[0,1)$ be given. To study how solutions to (A) change when the initial conditions vary, we specify $y_{0}>y^{R}\left(a_{0}\right)$. Let $a \equiv\left\{a_{0}, \ldots, a_{N}\right\}$ and $y \equiv\left\{y_{0}, \ldots, y_{N}\right\}$ be the sequences that satisfy

$$
\int_{a_{i}}^{a_{i+1}} U_{1}^{R}\left(y_{i}, m\right) f(m) d m=0 \quad(i=0, \ldots, N-1),
$$

and

$$
U^{S}\left(y_{i}, a_{i}, b\right)-U^{S}\left(y_{i-1}, a_{i}, b\right)=0, \quad(i=1, \ldots, N-1) .
$$

Given $a_{i}$, (26) determines $a_{i+1}$ as a function of $y_{i}$; given $y_{i-1}$, (27) determines $y_{i}$ as a function of $a_{i}$. Totally differentiating (26) with respect to $y_{i}$ and (27) with respect to $a_{i}$ yields

$$
\begin{aligned}
& -\int_{a_{i}}^{a_{i+1}} U_{11}^{R}\left(y_{i}, m\right) f(m) d m \\
& \quad \equiv U_{1}^{R}\left(y_{i}, a_{i+1}\right) f\left(a_{i+1}\right) v_{i}-U_{1}^{R}\left(y_{i}, a_{i}\right) f\left(a_{i}\right) w_{i}^{-1} \\
& \quad(i=0, \ldots, N-1),
\end{aligned}
$$

and

$$
\begin{array}{r}
U_{2}^{S}\left(y_{i}, a_{i}, b\right)-U_{2}^{S}\left(y_{i-1}, a_{i}, b\right) \equiv U_{1}^{S}\left(y_{i-1}, a_{i}, b\right) v_{i-1}^{-1}-U_{1}^{S}\left(y_{i}, a_{i}, b\right) w_{i} \\
(i=1, \ldots, N-1),
\end{array}
$$

where $v_{i} \equiv d a_{i+1} / d y_{i}(i=0, \ldots, N-1), w_{0}^{-1} \equiv 0$, and $w_{i} \equiv d y_{i} / d a_{i} \quad(i=1$, $\ldots, N-1)$. For fixed $a_{0}$, an initial specification of $y_{0}$ determines

$$
v_{0}=-\int_{a_{0}}^{a_{1}} U_{11}^{R}\left(y_{0}, m\right) f(m) d m / U_{1}^{R}\left(y_{0}, a_{1}\right) f\left(a_{1}\right),
$$

and then (28) and (29) determine $v_{i}$ and $w_{i}$ for $i=1, \ldots, N-1$. Since $d a_{i+1} / d a_{1}$ is given by $\prod_{j=1}^{i} w_{j} v_{j}$, to prove the Theorem it suffices to show that $w_{i} \geq 1$ and $v_{i} \geq 1$ for $i=1, \ldots, N-1$. 
First, $v_{0} \geq 1$, since

$$
\begin{aligned}
-\int_{a_{0}}^{a_{1}} U_{11}^{R}\left(y_{0}, m\right) f(m) d m \equiv & -\int_{a_{0}}^{a_{1}} U_{11}^{R}\left(y_{0}, m\right) f(m) d m \\
& +\int_{0}^{a_{0}} U_{11}^{R}\left(y_{0}, m\right) f(m) d m \\
\geq & U_{1}^{R}\left(y_{0}, a_{1}\right) f\left(a_{1}\right)-U_{1}^{R}\left(y_{0}, a_{0}\right) f\left(a_{0}\right) \\
\geq & U_{1}^{R}\left(y_{0}, a_{1}\right) f\left(a_{1}\right)>0 .
\end{aligned}
$$

The first inequality in (30) follows by hypothesis, since $a_{1}>a_{0}$, while the second and third follow from (26), since $U_{11}^{R}(\cdot)<0, U_{12}^{R}(\cdot)>0$, and $a_{1}>a_{0}$. The proof now follows by induction, for if $v_{i-1} \geq 1$ then $w_{i} \geq 1$ by (29), the fact that $y_{i}>y_{i-1}$, Lemma 2 , and the hypothesis that $U_{2}^{S}(y, a, b)+U_{1}^{S}(y, a, b)$ is nondecreasing in $y$. If $w_{i} \geq 1$, then $v_{i} \geq 1$ follows by a similar argument from (28). Q.E.D.

Remarks: The conditions of Theorem 2 are met by our example. They also hold for more general specifications. For example, if $F(m)$ is uniform (on $[0,1])$ and, for $i=R, S, U^{i}(\cdot)$ depends on $y$ and $m$ only through $y-m$ (that is, if there exist concave functions $\bar{U}^{i}$ such that $U^{S}(y, m, b) \equiv \bar{U}^{S}(y-m, b)$ and $U^{R}(y, m)$ $\left.\equiv \bar{U}^{R}(y-m)\right)$, then the functions required by the hypotheses of Theorem 2 to be nondecreasing and nonincreasing are both constant. Thus, (M) is guaranteed to hold if, after $m$ is rescaled to make $F(m)$ uniform (which can be done without affecting the signs of the $U_{11}^{i}(\cdot)$ and $\left.U_{12}^{i}(\cdot)\right)$, each player's preferences shift uniformly with $m$. It is also clear from the proof that the hypotheses of Theorem 2 are significantly stronger than (M): the proof established that an increase in $a_{1}$ leads to larger increases in all subsequent $a_{i}$, but all that (M) really requires is that all of the subsequent $a_{i}$ increase. Since (M) is, in turn, only a sufficient condition for the comparative statics results that follow, the hypotheses of Theorem 2 are quite far from being necessary for the comparative statics results to hold.

We shall now pause to establish a few useful lemmas.

LEMMA 3: For a given value of $b$, if $1 \leq N \leq N(b)$, there is exactly one partition equilibrium of size $N$. Further, if $a(N, b)$ and $a\left(N^{\prime}, b\right)$ are two equilibrium partitions for the same value of $b$, and if $N^{\prime}=N+1$, then $a_{i-1}(N, b)<a_{i}\left(N^{\prime}, b\right)<a_{i}(N, b)$ for all $i=1, \ldots, N$.

Proof: The first statement is an immediate consequence of Theorem 1 and assumption (M). That $a_{i}\left(N^{\prime}, b\right)<a_{i}(N, b)$ follows because if $a_{i}\left(N^{\prime}, b\right) \geq a_{i}(N, b)$ for some $i=1, \ldots, N$, then $a_{1}\left(N^{\prime}, b\right) \geq a_{1}(N, b)$ by (M). This leads to a contra- 
diction of $a_{N^{\prime}}\left(N^{\prime}, b\right)=a_{N}(N, b)=1$. That $a_{i-1}(N, b)<a_{i}\left(N^{\prime}, b\right)$ follows from $\left(\mathbf{M}^{\prime}\right)$ by a similar argument.

Q.E.D.

Lemma 4 says that if two partial partitions have the same endpoints, the partition associated with agents' preferences closer together begins with larger steps. This follows because the rate of increase of step size increases as preferences diverge. That is, if $a_{i-1}<a_{i}$ are fixed and $b>b^{\prime}$, then whenever $a_{i+1}(b)$ and $a_{i+1}\left(b^{\prime}\right)>a_{i}$ satisfy

$$
V\left(a_{i-1}, a_{i}, a_{i+1}(b), b\right)=V\left(a_{i-1}, a_{i}, a_{i+1}\left(b^{\prime}\right), b^{\prime}\right)=0,
$$

then $a_{i+1}(b)>a_{i+1}\left(b^{\prime}\right)$.

Lemma 4: If $a(K, b)$ and $a\left(K, b^{\prime}\right)$ are two partial partitions of length $K$ satisfying (A) with $b^{\prime}<b$, and $a_{0}(K, b)=a_{0}\left(K, b^{\prime}\right)=0$, then $a_{K}(K, b)=a_{K}\left(K, b^{\prime}\right)$ implies that $a_{i}(K, b)<a_{i}\left(K, b^{\prime}\right)$ for all $i=1, \ldots, K-1$.

Proof: The proof is by induction on $K$. For $K=1$, the Lemma is vacuously true. Suppose that $K>1$ and that the conclusion of the Lemma is true for all $i=1, \ldots, K-1$. Fix $b>b^{\prime}$, and let $a(K, b)$ and $a\left(K, b^{\prime}\right)$ be as in the statement of the Lemma. Suppose by way of contradiction that $a_{j}(K, b) \geq a_{j}\left(K, b^{\prime}\right)$ for some $j$ such that $0<j<K$; suppose further that $j$ is the largest index less than $K$ such that this inequality is satisfied, so that $a_{i}(K, b)<a_{i}\left(K, b^{\prime}\right)$ for all $i$ such that $j<i<K$. Let ${ }^{x} a \equiv\left({ }^{x} a_{0},{ }^{x} a_{1}, \ldots,{ }^{x} a_{j}\right)$ be the partial partition that satisfies $V\left({ }^{x} a_{i-1},{ }^{x} a_{i},{ }^{x} a_{i+1}, b^{\prime}\right)=0$ for $i=1, \ldots, j-1$ with ${ }^{x} a_{0}=0$ and ${ }^{x} a_{1}=x$. Since $a_{j}\left(K, b^{\prime}\right)={ }^{a_{1}\left(K, b^{\prime}\right)} a_{j}$ and, by assumption, $a_{j}(K, b)>a_{j}\left(K, b^{\prime}\right)$, it follows from (M) and the continuity of ${ }^{x} a$ in $x$ that there is an $\bar{x} \geq a_{1}\left(K, b^{\prime}\right)$ such that $a_{j}(K, b)={ }^{\bar{x}} a_{j}$, and that ${ }^{\bar{x}} a_{i} \geq a_{i}\left(K, b^{\prime}\right)$ for $1 \leq i \leq j$. Let ${ }^{\bar{x}} a \equiv \bar{a}$. We can establish the following relationships:

$$
\begin{aligned}
V\left(\bar{a}_{j-1}, a_{j}(K, b), a_{j+1}(K, b), b\right) & \geq V\left(\bar{a}_{j-1}, a_{j}(K, b), a_{j+1}\left(K, b^{\prime}\right), b\right) \\
& >V\left(\bar{a}_{j-1}, a_{j}(K, b), a_{j+1}\left(K, b^{\prime}\right), b^{\prime}\right) \\
& =V\left(\bar{a}_{j-1}, \bar{a}_{j}, a_{j+1}\left(K, b^{\prime}\right), b^{\prime}\right) \geq 0 .
\end{aligned}
$$

The first step follows because $a_{j+1}(K, b)<a_{j+1}\left(K, b^{\prime}\right)$ and $U^{S}\left(\bar{y}\left(a_{j}(K, b), a\right)\right.$, $\left.a_{j}(K, b), b\right)$ is decreasing in $a$ for $a \geq a_{j+1}(K, b)$ by Lemma 2; the second step follows because $V(\cdot, b)>V\left(\cdot, b^{\prime}\right)$; the third step follows because $a_{j}(K, b)=$ $\bar{a}_{j}$. To verify the final inequality first observe that $U^{S}\left(\bar{y}\left(\bar{a}_{j-1}, \bar{a}_{j}\right), \bar{a}_{j}, b^{\prime}\right)<$ $U^{S}\left(y^{R}\left(\bar{a}_{j}\right), \bar{a}_{j}, b^{\prime}\right)$, since $U^{S}\left(y, \bar{a}_{j}, b^{\prime}\right)$ is increasing in $y$ for $y \leq y^{S}\left(\bar{a}_{j}, b\right)$ and $\bar{y}\left(\bar{a}_{j-1}, \bar{a}_{j}\right)<y^{R}\left(\bar{a}_{j}\right)<y^{S}\left(\bar{a}_{j}\right)$; this implies that either $V\left(\bar{a}_{j-1}, \bar{a}_{j}, \bar{a}_{j}, b^{\prime}\right)>0$ and so $V\left(\bar{a}_{j-1}, \bar{a}_{j}, a, b^{\prime}\right)>0$ for all $a \in\left[\bar{a}_{j}, 1\right]$ or there is a unique $\bar{a}_{j+1} \in\left(\bar{a}_{j}, 1\right]$ such that $V\left(\bar{a}_{j-1}, \bar{a}_{j}, \bar{a}_{j+1}, b^{\prime}\right)=0$ and $V\left(\bar{a}_{j-1}, \bar{a}_{j}, a, b^{\prime}\right) \geq 0$ for $a \in\left[\bar{a}_{j}, \bar{a}_{j+1}\right]$. If $\bar{a}_{j+1}$ exists, it follows from the construction of $\bar{a}$ that $\bar{a}_{j+1} \geq a_{j+1}\left(K, b^{\prime}\right)>a_{j+1}(K, b)$. On the other hand, since $\bar{a}_{j}=a_{j}(K, b)$, the induction hypothesis ensures that $\bar{a}_{j-1}$ 
$\geq a_{j-1}(K, b)$, and $V\left(a_{j-1}(K, b), a_{j}(K, b), a_{j+1}(K, b), b\right)=0$ by construction. But then Lemma 2 implies that $V\left(\bar{a}_{j-1}, a_{j}(K, b), a_{j+1}(K, b), b\right)<0$, contradicting (31) and establishing the Lemma.

LEMMA 5: If $a(K, b)$ and $a\left(K, b^{\prime}\right)$ are two partial partitions of length $K$ satisfying (A) with $b>b^{\prime}$ and $a_{0}(K, b)=a_{0}\left(K, b^{\prime}\right)=0$, then $a_{1}(K, b)=a_{1}\left(K, b^{\prime}\right)$ implies that $a_{i}(K, b)>a_{i}\left(K, b^{\prime}\right)$ for all $i=2, \ldots, K$.

Lemma 5 is an immediate consequence of Lemma 4 and assumption (M). Therefore the proof is omitted.

LEMMA 6: The maximum possible equilibrium partition size, $N(b)$, is nonincreasing in $b$, the difference between agents' preferences.

Proof: Suppose $b^{\prime}<b$. Let $a(N(b), b)$ be a partition equilibrium of size $N(b)$, and let $\bar{a}\left(N(b), b^{\prime}\right)$ be the partial partition satisfying (A) with $\bar{a}_{1}\left(N(b), b^{\prime}\right)$ $=a_{1}(N(b), b)$. By Lemma $5, \bar{a}_{i}\left(N(b), b^{\prime}\right)<a_{i}(N(b), b)$ for all $i=2, \ldots, N(b)$. In particular, $\bar{a}\left(N(b), b^{\prime}\right)$ is at least of length $N(b)$. It follows that $N\left(b^{\prime}\right) \geq N(b)$. Q.E.D.

We are now ready to generalize the comparative statics results of Section 4 .

THEOREM 3: For given preferences (i.e., b), $R$ always strictly prefers equilibrium partitions with more steps (larger $N$ 's).

ReMARK: Since $R$ bases his choice of $y$ on rational expectations and $F$ is fixed, the Theorem extends the argument of Section 4 that equilibria with more steps are, ceteris paribus, more informative. A similar comment applies to Theorem 4 below, in connection with changes in $b$.

Proof: Fix $b$, and let $a(N)$ be a partition equilibrium of size $N<N(b)$. We shall argue that $a(N)$ can be continuously deformed to the (unique) partition equilibrium of size $N+1$, increasing the expected utility of $R$, denoted $E U^{R}$, throughout the deformation.

Let $a^{x} \equiv\left(a_{0}^{x}, a_{1}^{x}, \ldots, a_{N+1}^{x}\right)$ be the partition that satisfies (A) for $i=$ $2, \ldots, N$ with $a_{0}^{x}=0, a_{N}^{x}=x$, and $a_{N+1}^{x}=1$. If $x=a_{N-1}(N)$ then $a_{1}^{x}=0$, and if $x=a_{N}(N+1)$ then $a^{x}=a(N+1)$ and (A) is satisfied for all $i=1, \ldots, N$. When $x \in\left[a_{N-1}(N), a_{N}(N+1)\right]$, which is a nondegenerate interval by Lemma 3 , $E U^{R}(x)$ is strictly increasing in $x$. To see this, note first that $V\left(c, a_{1}^{x}, a_{2}^{x}, b\right)$ $\neq 0$ for all $c \in\left[0, a_{1}^{x}\right]$ if $x \in\left[a_{N-1}(N), a_{N}(N+1)\right)$. This follows because $\left(a_{N+1}(N+1), a_{N+1}(N), \ldots, a_{N+1}(1), a_{N+1}(0)\right)$ is a backward solution of (A) of length $N+1$, and $\left(\mathbf{M}^{\prime}\right)$ guarantees that any other backward solution of (A), $a$, of length $N+1$ with $a_{0}=1$ and $a_{1}=x$ must satisfy $x>a_{N+1}(N)$. Moreover $V(0$, $\left.a_{1}(N+1), a_{2}(N+1), b\right)=0$ by the definition of $a(N+1)$, and hence $-V(c$, $\left.a_{1}(N+1), a_{2}(N+1), b\right)>0$ for all $c \in\left(0, a_{1}(N+1)\right]$ by Lemma 2 . It follows 
from the continuity of $V$ with respect to $x$ that

$$
\begin{aligned}
& -V\left(c, a_{1}^{x}, a_{2}^{x}, b\right) \equiv U^{S}\left(\bar{y}\left(c, a_{1}^{x}\right), a_{1}^{x}, b\right)-U^{S}\left(\bar{y}\left(a_{1}^{x}, a_{2}^{x}\right), a_{1}^{x}, b\right)>0 \\
& \text { for all } x \in\left[a_{N-1}(N), a_{N}(N+1)\right) \text { and } c \in\left[0, a_{1}^{x}\right] .
\end{aligned}
$$

Now $E U^{R}(x)$ is given by

$$
E U^{R}(x) \equiv \sum_{j=1}^{N+1} \int_{a_{j-1}^{x}}^{a_{j}^{x}} U^{R}\left(\bar{y}\left(a_{j-1}^{x}, a_{j}^{x}\right), m\right) f(m) d m .
$$

Since $\bar{y}\left(a_{j-1}^{x}, a_{j}^{x}\right)$, defined in (9) as $R$ 's best response to a signal in the step $\left[a_{j-1}^{x}, a_{j}^{x}\right]$, maximizes the $j$ th term in the sum and since $a_{N+1}^{x} \equiv 1$, the Envelope Theorem yields

$$
\begin{aligned}
\frac{d E U^{R}(x)}{d x} \equiv \sum_{j=1}^{N} f\left(a_{j}^{x}\right) \frac{d a_{j}^{x}}{d x}[ & U^{R}\left(\bar{y}\left(a_{j-1}^{x}, a_{j}^{x}\right), a_{j}^{x}\right) \\
& \left.-U^{R}\left(\bar{y}\left(a_{j}^{x}, a_{j+1}^{x}\right), a_{j}^{x}\right)\right] .
\end{aligned}
$$

Assumption (M) guarantees that $d a_{j}^{x} / d x>0$ for all $j=1, \ldots, N$, and

$$
\begin{aligned}
& U^{R}\left(\bar{y}\left(a_{j-1}^{x}, a_{j}^{x}\right), a_{j}^{x}\right)-U^{R}\left(\bar{y}\left(a_{j}^{x}, a_{j+1}^{x}\right), a_{j}^{x}\right) \\
& \quad \geq U^{S}\left(\bar{y}\left(a_{j-1}^{x}, a_{j}^{x}\right), a_{j}^{x}, b\right)-U^{S}\left(\bar{y}\left(a_{j}^{x}, a_{j+1}^{x}\right), a_{j}^{x}, b\right) \geq 0 \\
& \quad(j=1, \ldots, N) .
\end{aligned}
$$

The first inequality in (35) holds because $\bar{y}\left(a_{j-1}^{x}, a_{j}^{x}\right)<\bar{y}\left(a_{j}^{x}, a_{j+1}^{x}\right)$ and $U_{13}^{S}(\cdot)$ $>0$; the second inequality is an equality for $j=2, \ldots, N$ by (A) and the definition of $a^{x}$, and holds strictly for $j=1$ by (32). This establishes the Theorem. Q.E.D.

THEOREM 4: For a given number of steps (i.e., $N$ ), $R$ always prefers the equilibrium partition associated with more similar preferences (i.e., a smaller value of $b$ ).

The proof of Theorem 4 is a straightforward application of Lemma 5 and an argument like that used to prove Theorem 3 , and is therefore omitted.

THEOREM 5: For given preferences (i.e., $b$ ), $S$ always strictly prefers ex ante (that is, before learning his type) equilibrium partitions with more steps (larger $N$ 's).

Proof: Maintaining the notation used in the proof of Theorem 3,

$$
E U^{S}(x) \equiv \sum_{j=1}^{N+1} \int_{a_{j-1}^{x}}^{a_{j}^{x}} U^{S}\left(\bar{y}\left(a_{j-1}^{x}, a_{j}^{x}\right), m, b\right) f(m) d m .
$$


It follows that

$$
\begin{aligned}
\frac{d E U^{S}(x)}{d x} \equiv \sum_{j=1}^{N} f\left(a_{j}^{x}\right) \frac{d a_{j}^{x}}{d x}\left[U^{S}\left(\bar{y}\left(a_{j-1}^{x}, a_{j}^{x}\right), a_{j}^{x}, b\right)\right. & \left.-U^{S}\left(\bar{y}\left(a_{j}^{x}, a_{j+1}^{x}\right), a_{j}^{x}, b\right)\right] \\
+ & \sum_{j=1}^{N+1} \frac{d \bar{y}\left(a_{j-1}^{x}, a_{j}^{x}\right)}{d x} \int_{a_{j-1}^{x}}^{a_{j}^{x}} U_{1}^{S}\left(\bar{y}\left(a_{j-1}^{x}, a_{j}^{x}\right), m, b\right) f(m) d m .
\end{aligned}
$$

The first term on the right-hand side of (37) is positive by (A), (32), and the definition of $a^{x}$. The second term is nonnegative since $d \bar{y}\left(a_{j-1}^{x}, a_{j}^{x}\right) / d x>0$ by (M), and the integral expressions are all nonnegative by our assumption that $U_{13}^{S}(\cdot)>0$ and by the first-order conditions that determine $R$ 's optimal choice of the $\bar{y}\left(a_{j-1}^{x}, a_{j}^{x}\right)$.

Q.E.D.

\section{CONCLUSION}

This paper represents an attempt to characterize rational behavior in interactive two-person situations where direct communication between agents is a possibility. While we have considered explicitly only a small subset of the universe of possible models with this property, our results can be generalized immediately beyond the confines of our model in several directions. These results hint that there may be a good case for presuming that direct communication is more likely to play an important role, the more closely related are agents' goals. Other interesting conclusions suggested by our theory are that perfect communication is not to be expected in general unless agents' interests completely coincide, and that once interests diverge by a given, "finite" amount, only no communication is consistent with rational behavior.

Some worthwhile extensions of the model are suggested by the fact that the structure of our model interacts with the rational-expectations character of our solution concept in such a way that concepts like lying, credibility, and credulity -all essential features of strategic communication-do not have fully satisfactory operational meanings within the model. Generalizations that would test the robustness of our results and help to remedy this defect include allowing lying to have costs for $S$, uncertain to $R$, in addition to those inherent in its effect on $R$ 's choice of action; allowing $R$ to be uncertain about $S$ 's preferences, and therefore about his incentives to communicate truthfully; and allowing $S$ to be uncertain about $R$ 's ability to check the accuracy of what he is told.

\section{University of California, San Diego}




\section{REFERENCES}

[1] Chatterjee, Kalyan, and William Samuelson: "The Simple Economics of Bargaining," Boston University, School of Management, Working Paper 2/81, 1979, revised 1981.

[2] Crawford, Vincent P.: “A Theory of Disagreement in Bargaining," Econometrica, 50(1982), 607-638.

[3] Green, Jerry, and Nancy Stokey: "A Two-Person Game of Information Transmission," Harvard University, H.I.E.R. Discussion Paper 751, March, 1980.

[4] Harsanyi, John C.: "Games with Incomplete Information Played by 'Bayesian' Players II: Bayesian Equilibrium Points," Management Science, 14(1968), 320-334.

[5] — Rational Behavior and Bargaining Equilibrium in Games and Social Situations. London and New York: Cambridge University Press, 1977.

[6] Holmström, Bengt: "On the Theory of Delegation," mimeo, Northwestern University, June, 1980.

[7] KRePS, David M., ANd Robert Wilson: "Reputation and Imperfect Information," Journal of Economic Theory, 27(1982), 253-279.

[8] LoÈve, Michel: Probability Theory. New York: D. Van Nostrand Company, Inc., 1955.

[9] Milgrom, Paul, and John Roberts: "Limit Pricing and Entry under Incomplete Information: an Equilibrium Analysis," Econometrica, 50(1982), 443-460.

[10] - "Predation, Reputation, and Entry Deterrence," Journal of Economic Theory, 27(1982), $280-312$.

[11] Milgrom, Paul, and Robert Weber: "Distributional Strategies for Games with Incomplete Information," Mathematics of Operations Research, forthcoming.

[12] NovsheK, William, and Hugo Sonnenschein: "Fulfilled Expectations Cournot Duopoly with Information Acquisition and Release," Bell Journal of Economics, 13(1982), 214-218.

[13] Schelling, Thomas C.: The Strategy of Conflict. London and New York: Oxford University Press, 1963.

[14] Sobel, Joel, and IChiro Takahashi: “A Multi-Stage Model of Bargaining,” U.C.S.D. Discussion Paper 80-25*, 1980, revised 1982.

[15] SPENCE, MichaEL: “Job Market Signaling," Quarterly Journal of Economics, 87(1973), 355-374. 